\title{
Periconceptional overnutrition and offspring gender influence the development of adiposity and adipogenic gene expression in the lamb
}

\author{
Leewen Rattanatray ${ }^{1}$, Severence MacLaughlin ${ }^{2}$, Beverly Muhlhausler ${ }^{2}$, Dave Kleemann ${ }^{3}$, \\ Simon Walker ${ }^{3}$ and I. Caroline McMillen ${ }^{1}$ \\ ${ }^{1}$ School of Molecular and Biomedical Sciences, Adelaide University, Adelaide, South Australia, Australia, ${ }^{2}$ SANSOM \\ Institute, University of South Australia, Adelaide, South Australia, Australia and ${ }^{3}$ South Australian Research and \\ Development Institute, Rosedale, South Australia, Australia
}

Maternal obesity is associated with an increased risk of gestational diabetes in the mother and an increased birth weight and fat mass in the infant ${ }^{(1,2)}$. Being of high birth weight increases the risk of becoming overweight or obese in child and adult life ${ }^{(3)}$. It is not known, however, whether maternal obesity before and immediately after conception is associated with an increased risk of obesity in the offspring, and if so, whether the impact of maternal obesity can be ameliorated by maternal weight loss immediately before conception.

The present study will determine whether entering pregnancy obese leads to the programming of offspring obesity and assess the impact of prepregnancy dietary restriction on the fat mass and the expression of genes that regulate adipogenesis and lipogenesis in the offspring at 4 months of age in the sheep. It is hypothesized that periconceptional obesity will result in increased expression of adipogeneic and lipogenic genes and increased fat mass in the postnatal lamb.

Donor ewes (n 23) were randomly assigned to one of four treatment groups, either control-control (C-C), control-restricted (C-R), high-high $(\mathrm{H}-\mathrm{H})$ or high-restricted $(\mathrm{H}-\mathrm{R})$ nutritional treatment groups. C-C ewes $(n$ 6) were maintained at $100 \%$ metabolisable energy requirements (MER) for 4 months before conception. The C-R ewes ( $n$ 6) were maintained at $100 \%$ MER for the first 3 months, then 1 month before conception were placed on a dietary restriction of 70\% MER. The H-H ewes ( $n$ 6), were fed ad libitum (170-190\% MER) for 4 months before conception. H-R ewes ( $n$ 5) were fed ad libitum (170-190\% MER) for 3 months, and then 1 month before conception were placed on an energy-restricted diet of $70 \%$ MER. The reproductive cycle of all experimental ewes was synchronized and super ovulation was induced. Donor ewes were artificially inseminated by laparoscopy. Embryos were collected and transferred to synchronized recipient ewes maintained on a control diet (100\% MER) at embryonic day 6-7. All ewes were allowed to give birth naturally at term, and lambs remained with their mothers after birth until weaning at 3 months of age. Birth weights and weights at post mortem were recorded at 4 months of age $(n 41)$. At post mortem subcutaneous, perirenal and omental fat depots were weighed and collected and relative expression of adiponectin, leptin, lipoprotein lipase (LPL) and glycerol-3-phosphate dehydrogenase (G3PDH) were determined by quantitative RT PCR.

There was no effect of periconceptional nutrition on offspring birth weight or post-mortem weight at 4 months of age. Birth weight was higher in male offspring compared with female offspring. Male lambs were significantly heavier than females at 4 months of age $(P<0.001)$. There was no effect of periconceptional nutrition on subcutaneous, perirenal and omental fat mass. However, periconceptional overnutrition increased total fat mass in the female lambs at 4 months of age and the dietary restriction in the overnourished ewes albated this effect. Females irrespective of treatment group had a significant increase in relative omental $(P<0.001)$, subcutaneous $(P<0.001)$ and perirenal fat mass $(P=0.013)$. There was no effect of periconceptional nutrition on the expression of adiponectin, leptin, LPL or G3PDH in the subcutaneous adipose depot. The expression of G3PDH in subcutaneous adipose tissue was higher in females compared with males $(P=0.042)$ and females also tended to express more leptin $(P=0.072)$.

It has been demonstrated that maternal obesity during the periconceptual period is associated with an increase in total fat mass in the female offspring at 4 months of age. These data suggest that maternal dietary restriction in the month before conception is effective in preventing this increased accumulation of fat mass in the offspring. These findings suggest that the effects of periconceptual overnutrition on the early embryo may alter the subsequent development of adipose tissue, and the impact of prepregnancy maternal obesity may be reduced by a reduction in maternal energy intake in the period immediately preceding conception.

1. Catalano PM, Kirwan JP, Haugel-de Mouzonz S \& Kingzz J (2003) J Nutr 133, 1674S-1683S

2. Gillman MW, Rifas-Shiman S, Berkey CS, Field AE \& Colditz GA (2003) Pediatrics 111, 221-226.

3. Parsons TJ, Power C \& Manor O (2001) Br Med J 323, 1331-1335. 